\title{
Analysis of the risks and countermeasures of enterprise employee recruitment
}

\author{
Danwen Chen ${ }^{1, a}$ and Xi Zhou ${ }^{2, b}$ \\ ${ }^{1}$ School of Education, Jianghan University, Sanjiaohu Road 8, Wuhan Economic \& Technological \\ Development Zone, Wuhan, Hubei, China \\ ${ }^{2}$ School of Education, Jianghan University, Sanjiaohu Road 8, Wuhan Economic \& Technological \\ Development Zone, Wuhan, Hubei, China \\ acdw@jhun.edu.cn, ${ }^{\mathrm{b}} 649613327 @ q q . c o m$
}

Keywords: recruitment, risks, solution

\begin{abstract}
The aim of this paper was to investigate the risks of and solution to the recruitment process in China's enterprises. A survey on structure of human resources and recruitment system of an enterprise in China was conducted. The results indicate a lot of underlying problems: the recruitment cycle was rather long; the actual recruitment cycle was longer than expected; the feedback was slowly given because interviewer's heavy workload and indifferent attitudes, resulting in a loss of talents; a quarter of "VIP" employees, which refer to employees recruited though the back door, were misgoverned; information asymmetry caused the enterprise to recruited people who behave good in interview but bad in work. The author concluded that enterprises should pay more attention to the quality of recruitment process and the speed of message replying, establish a harmonious and fair work atmosphere, strengthen the relationship and enhance mutual understanding among employees, make the full use of back-door employees, improve work handover and induction training and apply multiple talent assessment methods to comprehensive assess candidates.
\end{abstract}

\section{The risk of recruitment risks and its significance}

The risk of recruitment refers to the flaws in the recruitment process, which manifests as the outflow of talents or decision mistakes and results in a loss in time, manpower and revenue. The risk of recruitment is objective, uncertain and controllable.

Along with the arrival and development of "knowledge economy", manpower has played a more and more important role in competition among enterprises. Human resources have become the core competitiveness, affecting the rise and fall of enterprises. The risk of recruitment has long been a major problem in the recruitment process Although unable to completely avoid recruitment risk, enterprises can control and reduce the risk of recruitment through a variety of methods because of the risk's controllability. Studying on the risk of recruitment can help repairing vulnerabilities, improving the recruitment process, saving cost and hiring the appropriate talent, all of which will not only bring new thinking for the enterprise, but directly affect the rise and fall as well.

\section{2 case study}

\subsection{Introduction}

This paper studied on a Sino-American joint auto parts manufacturer based in Wuhan, the subsidiaries of which are located in major cities like Guangzhou, Xiangyang, Shiyan. The manufacturer has more than 10 thousand employees in total and sells its products all over the world. The author focused on the recruitment in Wuhan, because the headquarter in Wuhan has set up not only an almost complete management system and organizational structure but a clear division of 
labor as well.

2.2 the risk of recruitment cycle

Table 1. recruitment cycle of the manufacturer (2013-2015)

\begin{tabular}{|c|c|c|c|}
\hline year & position & $\begin{array}{c}\text { Expected recruitment } \\
\text { cycle(days) }\end{array}$ & $\begin{array}{c}\text { Actual recruitment } \\
\text { cycle(days) }\end{array}$ \\
\hline \multirow{2}{*}{2013} & Administrative staff & 90 & 105 \\
\cline { 2 - 4 } & Operational staff & 60 & 65 \\
\hline \multirow{2}{*}{2014} & Administrative staff & 90 & 112 \\
\cline { 2 - 4 } & Operational staff & 60 & 64 \\
\hline \multirow{2}{*}{2015} & Administrative staff & 90 & 100 \\
\cline { 2 - 4 } & Operational staff & 60 & 70 \\
\hline
\end{tabular}

According to table 1, the actual recruitment cycles in both positions are longer than expectation. Qualified candidates are hard to find in this crowded talent market, resulting in a long recruitment cycle and short of hands in needy departments. Especially, it takes a lot of time, human resources and financial resources to recruit employees in financial department and business development department, the former of which even spent 10 months before finding the appropriate employees.

\subsection{The risk of interviewee's waiting time}

The manufacturers' human resources department replies slowly. For many times the interviewers were having meeting during the time they ought to have interviewed the candidates, who were thus forced to wait for more than an hour. Once, a candidate had waited for 3 hours because of the aforementioned reason. In such a competitive talent market, qualified candidates tend to judge the quality of an employer through details. If not respected, candidates might change their view of the company. It may seem like a small thing to keep candidates waiting, but it also reflects whether an enterprise is people-oriented and is respectful to employees, whether an enterprise can be reliable and promising.

2.4 The risk of "VIP" employees

Table 2. ratio of "VIP” employees in each department (2015)

\begin{tabular}{|c|c|c|c|}
\hline department & No. of staff & No. of “VIP” & $\begin{array}{c}\text { "VIP” staff /total } \\
\text { staff }\end{array}$ \\
\hline Human resources & 4 & 3 & $75 \%$ \\
\hline finances & 6 & 1 & $16.7 \%$ \\
\hline $\begin{array}{c}\text { Business } \\
\text { development }\end{array}$ & 5 & 0 & 0 \\
\hline IT & 2 & 1 & $50 \%$ \\
\hline $\begin{array}{c}\text { General manager } \\
\text { office }\end{array}$ & 4 & 1 & $25 \%$ \\
\hline Purchase \& order & 2 & 0 & 0 \\
\hline Industry engineering & 6 & 0 & $50 \%$ \\
\hline $\begin{array}{c}\text { Project } \\
\text { Continuous } \\
\text { improvement }\end{array}$ & 2 & 1 & $25 \%$ \\
\hline Commerce & 4 & 1 & $50 \%$ \\
\hline $\begin{array}{c}\text { Party-masses } \\
\text { relationship }\end{array}$ & 2 & 1 & 0 \\
\hline
\end{tabular}

From table 2 the author discovered that the number of "VIP" employees (10) accounted for 1/4 of the total employees (40) in the auto parts manufacturer. "VIP" employees are usually seen in the 
readily developed large-scale enterprises. The research objective of this paper is a mature and promising sizeable manufacturer, so there exists the potentiality of "VIP" employees. It's understood that in two departments there is at least one "VIP" employee, and the number of "VIP" employees is positively correlated with the popularity of the department. For example, Human resources department has 3 "VIP" employees. Most of the "VIP" employees are recruited through internal staff recommendations or chief's arrangements. Because of their special background, "VIP" employees might influence the daily work of the department, the atmosphere of the enterprise, the relationship between employees and the external image of the enterprise. "VIP" employee's incapability might hinder daily operation, reduce efficiency or bring greater trouble to work. Besides, "VIP" employees affect other employees' perceptions of the business and reduces other employees' sense of belonging and collective honor. Because of their sensitive identity, the "VIP" employees will find themselves marginalized and hard to fit into the department. If "VIP" employees act in their own way and refuse to obey orders, the conflict between "VIP" and the other employees will only deteriorate and impair the working atmosphere.

\subsection{The risk of information asymmetry}

Information asymmetry in this study refers to that job seekers deliberately modify their resume to leave a falsely qualified impression on the interviewers; the enterprise, on the other hand, lacks the detailed information of the candidate and thus unable to make an accurate judgement. According to the survey, due to information asymmetric, the manufacturer failed to examine the candidates in financial department and engineering department. Both of the candidates deeply impressed interviewers with rich resume content, clear logic and strong communication skills shown in telephone interview. However, in the third round of interview led by recruitment department, the candidates' insufficient capacity betrayed their former performance. This kind of interviews are rich in resume and experienced in communication but poor in professional knowledge. If HR department fail to investigate and interview the candidates thoroughly, the enterprise will be easily deceived by false image created in resume, resulting in a loss in time and energy.

\section{Solutions to reduce recruitment risks}

3.1 Instilling the idea that human resources is the core competitiveness, urging and tracking the interviews , improving mutual communication and speeding up message reply

The company's financial position has long been vacant not only because of candidates' insufficiency in capabilities, but more due to the interviewer's indifference to recruitment. Nowadays the supply for personnel exceeds the demand. Facing a glut of resumes, interviewers began to be taken the recruitment work lightly. It seems that financial position is popular among candidates and does not suffer the shortage of interviewees, but recruitment is a two-way choice. Candidates are uneven in terms of their capacity, and truly qualified ones are to be missed if the enterprise neglect job seekers.

Therefore, both human resources department and recruitment department should attach more importance to enterprise's recruitment. Before recruitment department contacts the candidates and makes an appointment, human resources department should fully communicate with recruitment department to get acquaint with interviewer's daily schedule in recent days or recent weeks. Information shouldn't be given to the candidates until specific time of interview is settled (be sure to include alternative options). On the other hand, even if human resources department and recruitment departments agreed on all the details of a telephone interview, human resources department should track the entire interview and urge the recruitment department to punctually, preventing that interviewers postpone or forget the interview due to the heavy workload. Human resources department's supervision contributes to the objective of raising awareness of recruitment process in recruitment department and instilling the idea of human resources being the core 
competitiveness. If interviewer is caught by an emergency and can't show up on the interview, human resources department should immediately negotiate with interviewees and reach a mutual understanding. Although seemed trivial, such details are determinative factor of a company's reputation. Candidates judge whether a company is suitable through contacting with current employees, and their choice directly affect the company's future staff level.

\subsection{Treat "VIP” employees with an objective and dialectical manner}

There are 2 categories of "VIP" employees. The first category is those capable and attractive employees whose existence makes real contribution to the enterprise, so other employees will welcome them rather than resist them. The second category is those mediocre and stubborn employees who refuse to command and thus not only decrease efficiency but also disrupt harmony. But because of their powerful background, enterprise fails to criticize or dismiss them because many other party's interests are involved. However, treat "VIP" employees with an objective and dialectical manner turns disadvantage into advantage. The second category of "VIP" employees are strongly motivated, or in other words, very loyal to the company. In order not to be branded or marginalized, "VIP" employees tend to do more. Additionally, social networking is indispensable in nowadays society. "VIP" employees have broaden social circle which can be utilized by enterprise, and the way to a "VIP" employee's social network is through his heart. Therefore, the author gave following suggestions to treat "VIP" employees in a right way:

First, enterprise should establish a harmonious and impartial working atmosphere and boost understanding and trust between employees.

Solidarity, cooperation and coherence are necessary ingredient of an excellent enterprise. Creating a fair and harmonious atmosphere while setting up an impartial management system can help the employees to develop the sense of belonging, enhance awareness of cooperation, strengthen interdepartmental communication, improve work efficiency and maintain such working environment. Impartiality inside a company make employees snobby and cliquey. If an enterprise ignores employee's query and discontent when arranging "VIP" staff, dissatisfaction is sure to burst out from other staff work, hindering both the effective communication and the working process. Therefore, the implementation of a fair and equitable enterprise culture is necessary. Strictly executing the performance -related inspection system, equally treating all the employees and ensuring them that "No matter if it is a white cat or a black cat, a cat that can catch rats is a good cat", employees can then realize that they're running from the same starting line even though there are "VIP" employees and that they are assessed and promoted through the same standard. Before placing "VIP" employees in certain positions, enterprise should keep effective and respectful communication with department managers, discussing the importance of such placement rather than commanding the mangers to do so. Only in this way can department managers lead his team objectively and rationally instead of squeezing out "VIP" employees with the other staff.

Second, enterprise should make a comprehensive analysis of "VIP" employees and let everybody fully display his talents.

It's better for enterprises to make prior investigation about "VIP” employees' capacity and characteristics to assign suitable jobs for them according to their heterogeneity. Enterprises are responsible of creating a platform where employees can make the best of their ability and maximize the benefit for the company.

Third, enterprise should complete handover and induction training

When dealing with "VIP" employees whose background is too powerful to reject, what enterprises can do is to complete job handover and induction training. Induction training includes introduction to the enterprise and its operation system, code of business practice, work flow and professional knowledge. Employee's mastery of work flow, professional knowledge and code of business practice is of particular significance. The surrenderor should impart related skills step by step according to new staff's learning ability rather than teaching them once and letting them pondering by themselves for the sake of catching up on schedule. For those who do not understand 
the task and do not like to ask, managers need to guide them and cheer them on. Regular inspection and frequent meetings allows managers to promptly point out "VIP" employee's flaws, to help them straighten out working attitude, to understand their confusion, to solve their problems and to help "VIP" employees completing the conversion from "outsiders" to "insiders". Only with an appropriate job positioning and career planning can "VIP" employees quickly adjust their attitude, strengthen communication with colleagues, improve working efficiency and thus fit themselves into the whole enterprise.

3.3Adopt multiple talents assessment method to comprehensively analyze candidates and to prevent mistakenly recruit candidates lacking profession

China is wanting in a systematic and authorized talents assessment method, so enterprises can only modify the existing assessment methods according to the needs of themselves. The manufacturer in this research adopted the temperament type test scale in psychology in order to examine whether the candidate's personality is consistent with the corporate. But most candidates tend to optimize their image and the results didn't fully match the candidates' real personality, so the scale test results can only serve as a reference but not a determinative factor of interviewer's qualification. Therefore, the author gave the following suggestions to solve information asymmetry:

\subsubsection{Enterprise should strengthen the prior investigation of interviewers, establish a systematic talent pool and blacklist cheaters}

Resume consists of what interviewers want the interviewees to know, which means that working experiences and performance might be exaggerated. This requires recruiters to sharpen their eyes, judging the authenticity of the resume and digging out the hidden information according to their own recruitment experiences. For example, some candidates indicate that they are managers, but they keep from talking about how many subordinates they have and how's their performance. If recruiters can carefully select resumes, the probability of invalid recruitment will be greatly reduced.

\subsubsection{Enterprise should adopt multiple talent assessment method and add professional topics in written test}

The manufacturer's current talent assessment method consists of a written test and an interview, the former of which contains temperament type test, situation analysis and language translation while the latter of which is a one-to-one interview led by human resources department and recruitment department. According to the survey, the author discovered that interviewer's ignorance of examining interviewee's professional skills resulted in that the manufacturer didn't figure out candidate's incapacity until the third interview. The author then argues that human resources department should add a basic skills test to eliminate those with hollow resumes in the written test.

\section{4 conclusion}

Recruitment is an important process in human resources management, but it also brings about potential risks that are impossible to be totally prevented. Many reasons, such as the candidate's own problems, the interviewer's subjective judgment, the imperfection in recruitment process or the improper selection of recruitment channels, cause recruitment risks. Recruitment risks frustrate recruitment and harms the enterprise's benefits. However mature an enterprise is, it can hardly avoid recruitment risks. Only by exploring risks through self-examination and consistently improving recruitment process can enterprises effectively reduce risks and finally, attract talents in the competitive market. 


\section{References}

[1] Qing Huang. Risk exploration of human resource recruitment in large and medium sized private enterprises[J]. Enterprise Management. 2015(6):45-46

[2] Yan Li. Strategies for employers to cope with "information asymmetry"[J]. Consumption Economy,2012(05):49-51

[3] Mingli Shen. Research on risk management of enterprise human resource recruitment [J]. Industry and Technology Forum 2013(3):220-222

[4] Lin Wang. How to avoid recruitment risks[J].Human Resources. 2015(4):74-76

[5] Dingquan Yang, Xialing Li, Shaozhong Ye, Baochang Liang. Analysis of SME recruitment risks and their causes[J]. Enterprise Management. 2014(6):11-12

[6] $\mathrm{Lu} \mathrm{Li}$, Analysis of information asymmetry in recruitment[J]. Economists. 2014 (11) :238-239

[7] Jun Cao, Analysis and Countermeasures of Enterprise Recruitment Risk under the condition of information asymmetry[J]. Management discussion.2014(10): 54-55

[8] Ting Fan. Research on recruitment of small and medium sized enterprises in China -- Taking W company as an example

[9] George T Milkovich. Human Resource Management [M]. Mechanic industry Press， 2006

[10]Dhamijia,Pavitra.“E-recruitment:a roadmap towards human resource management.”Res World J Arts Sci Commer. 2012(3) 